\title{
Oxidación Electroquímica y Ozonización Aplicadas al Tratamiento de Aguas de Lavado de la Producción de Biodiesel
}

\author{
Kelly V. Patiño, Sandra M. Arroyave, Juan M. Marín \\ Grupo Procesos Fisicoquímicos Aplicados, Ciudad Universitaria, Calle 67 № 53-108, Universidad \\ de Antioquia, Medellín, Colombia (e-mail: jmmarin@udea.edu.co)
}

Recibido May. 05, 2011; Aceptado Jun. 28, 2011; Versión Final recibida Ago. 25, 2011

\begin{abstract}
Resumen
Se aplicaron tecnologías avanzadas de oxidación no fotoquímicas, ozonización y oxidación electroquímica, como posibles alternativas para tratar las aguas de lavado de biodiesel. La caracterización fisicoquímica de las aguas mostró altos niveles en demanda química y bioquímica de oxígeno, carbono orgánico total y metanol. Ambas tecnologías aumentaron levemente la biodegradabilidad del agua y mostraron valores de remoción similares, con remociones de demanda química de oxígeno entre 5 y $9 \%$ y de carbono orgánico total entre 9 y $11 \%$. Sin embargo la ozonización presenta características que la hacen más versátil para ser implementada a mayor escala como un sistema de tratamiento solo o acoplado a procesos biológicos, comúnmente usados para el tratamiento de aguas con alta carga orgánica.
\end{abstract}

Palabras clave: ozonización, oxidación electroquímica, biodiesel, aguas residuales, descontaminación

\section{Electrochemical Oxidation and Ozonation Applied to the Treatment of Wastewaters from Biodiesel Production}

\begin{abstract}
Non-photochemical advanced oxidation technologies, ozonation and electrochemical oxidation, as alternative technologies to treat wastewaters from biodiesel production were applied. The physicochemical characterization of the wastewaters showed high levels in biochemical and chemical oxygen demand, total organic carbon and residual methanol. Both technologies increased the wastewater biodegradability and showed similar efficiencies in the reduction of the organic loads, with chemical oxygen demand removal between 5 and $9 \%$ and total organic carbon removal between 9 and $11 \%$. However the ozonation showed to be a more versatile technology to be implemented on a larger scale as a system for treatment alone or coupled to biological processes that are typically used in treating waters with high organic load.
\end{abstract}

Keywords: ozonation, electrochemical oxidation, biodiesel, wastewater, decontamination 


\section{INTRODUCCIÓN}

En los últimos años, el Biodiesel se ha presentado como un combustible alternativo al diesel derivado del petróleo debido a su carácter renovable, a su buen desempeño y a que sus emisiones gaseosas son significativamente menores comparadas con las obtenidas cuando se usa el diesel tradicional. El método de fabricación más común es la transesterificación de aceite, mediante un catalizador alcalino, convirtiendo los ácidos grasos a ésteres metílicos en poco tiempo y con eficiencias del 99\% (Habib et al., 2010; Komers et al., 2010). Después de la reacción de transesterificación, a $60^{\circ} \mathrm{C}$ durante una hora, se obtiene biodiesel y glicerol, el cual se separa por decantación y se remueve. El biodiesel obtenido requiere purificación y para ello se lava con agua, la cual se emplea en una proporción de aproximadamente el $20 \%$ en volumen y es agregada en varias etapas, con períodos de decantación entre 12 y 24 horas luego de cada etapa (Berrios y Skelton, 2008). El agua de lavado constituye el agua residual proveniente del proceso de producción del biodiesel, la cual contiene grasas y aceites, catalizador básico, sales, jabones, trazas de glicerol y metanol. En consecuencia, el agua del lavado se debe someter a un proceso de tratamiento antes de ser devuelta al ambiente.

Los tratamientos reportados para este tipo de agua son pocos y entre ellos se encuentra un tratamiento biológico con Rhodotorula mucilaginosa a pH 6.8 y adición de nutrientes tales como nitrógeno (sulfato de amonio, cloruro de amonio o úrea), extracto de levadura, $\mathrm{KH}_{2} \mathrm{PO}_{4}$ y $\mathrm{MgSO}_{4} \cdot 7 \mathrm{H}_{2} \mathrm{O}$. Los resultados sugieren que éste tratamiento es útil para plantas de producción a pequeña escala, que la adición de úrea como fuente de nitrógeno facilita la degradación y que la relación óptima de C/N para el tratamiento se encuentra entre 17 y 68 (Suehara et al., 2005). Otro estudio realizó un tratamiento por medio de electrocoagulación usando electrodos de Aluminio, hierro y grafito en configuraciones Fe-Fe, Fe-C, Al-Al, Al-C y C-C. Los resultados muestran que las mejores condiciones se obtienen empleando ánodo de aluminio y cátodo de grafito con densidades de corriente entre 7.97 y $8.32 \mathrm{~mA} \mathrm{~cm}^{-2}$, tiempos de 20 a 25 minutos y un valor de $\mathrm{pH}$ de 6. La eficiencia de remoción en DQO alcanzada fue del $55.4 \%$ (Sirangsan et al., 2009). Las Tecnologías Avanzadas de Oxidación, TAOs, han sido ampliamente utilizadas para la degradación de contaminantes y destoxificación de aguas residuales que contienen compuestos recalcitrantes; algunas de ellas se caracterizan por la producción del radical hidroxilo $\left(\mathrm{OH}^{\circ}\right)$, el cual es un poderoso oxidante no selectivo, capaz de oxidar y mineralizar casi cualquier molécula orgánica produciendo $\mathrm{CO}_{2}$ y aniones inorgánicos (Domènech et al., 2004). Hincapié-Mejía et al. aplicaron las TAOs fotocatálisis heterogénea y foto-Fenton al tratamiento de aguas residuales de biodiesel. Los resultados mostraron que la fotocatálisis heterogénea no es una tecnologíaadecuada debido a las altas cargas orgánicas, mientras que la tecnología de foto-Fenton produjo remociones del $27.15 \%$ de COT y el $7.04 \%$ en DQO, empleando $0.3 \mathrm{mM}$ de hierro (II), $35 \mathrm{mM}$ de $\mathrm{H}_{2} \mathrm{O}_{2}$ y una lámpara con emisión máxima a 254 nm (Hincapié-Mejía et al., 2011).

La ozonización es una tecnología avanzada de oxidación, que no es exclusiva para la potabilización de agua, ya que tiene numerosas aplicaciones para el tratamiento de aguas residuales, debido a que mejora su biodegradabilidad haciendo más fácil el tratamiento biológico convencional. Las aplicaciones del ozono en tratamiento de aguas residuales, pueden agruparse en tres categorías, como desinfectante o biocida, oxidante clásico para remover contaminantes orgánicos, y pre o post-tratamiento de agentes para ayudar en otras operaciones unitarias como la coagulación, la floculación, la sedimentación, etc. (Beltrán, 2004).

Debido a su configuración electrónica, el ozono tiene diferentes tipos de reacciones en agua, formando radicales libres. Estos radicales libres, se propagan a través de mecanismos de pasos elementales para producir radicales hidroxilo, los cuales son extremadamente reactivos con cualquier especie orgánica y algunas especies inorgánicas, presentes en el agua. Por esta razón, las reacciones del ozono en agua, pueden ser clasificadas como directas e indirectas. Las reacciones directas son las verdaderas reacciones del ozono, que son las reacciones donde la molécula de ozono reacciona con especies químicas presentes en la matriz mientras que reacciones indirectas se realizan con el radical hidroxilo, formado de la descomposición del ozono de reacciones directas, con los compuestos presentes en el agua, por lo tanto, las reacciones 
directas del ozono (ecuaciones 1 y 2 ), son el paso inicial hacia las reacciones indirectas (ecuaciones 3 y 4), las cuales se muestran a continuación (Beltrán, 2004).

$$
\begin{aligned}
& 2 \mathrm{O}_{3}+\mathrm{H}_{2} \mathrm{O} \rightarrow 2 \mathrm{HO}^{\bullet}+2 \mathrm{O}_{2}+\mathrm{HO}_{2}^{\bullet} \\
& \mathrm{O}_{3}+\mathrm{HO}_{2}^{\bullet} \rightarrow \mathrm{HO}^{\bullet}+2 \mathrm{O}_{2} \\
& \mathrm{HO}^{\bullet}+\mathrm{HO}_{2}^{\bullet} \rightarrow \mathrm{H}_{2} \mathrm{O}+\mathrm{O}_{2} \\
& \mathrm{RH}+\mathrm{HO}^{\bullet}+\mathrm{H}_{2} \mathrm{O} \rightarrow \mathrm{ROH}+\mathrm{H}_{3} \mathrm{O}^{+} \rightarrow \text { productosoxidados }
\end{aligned}
$$

Los procesos biológicos son la opción más económica para eliminar contaminantes orgánicos, sin embargo, estos métodos tienen sus limitantes cuando las aguas presentan características de toxicidad contra los microorganismos usados en dichos procesos (Robinson et al., 2001). Algunos de estos procesos tienen aplicaciones limitadas en aguas con contenidos apreciables de iones inorgánicos, como lo mostraron Guillard y colaboradores, reportando que la eficiencia fotocatalítica del dióxido de titanio se reducía en presencia de aniones tales como $\mathrm{NO}_{3}{ }^{-}, \mathrm{Cl}^{-}, \mathrm{SO}_{4}{ }^{2-}, \mathrm{PO}_{4}{ }^{3-}$ (Guillard et al., 2005). Por el contrario, la presencia de dichos iones en el agua, incrementa la conductividad y por lo tanto las aplicaciones de tratamiento de aguas residuales basado en métodos de oxidación electroquímica resultan comúnmente favorecidas, especialmente los realizados con electrodo de diamante dopado con boro, el cual tiene la capacidad de mineralizar completamente los contaminantes orgánicos (Brillas et al., 2005), o transformarlos en subproductos biodegradables que pueden ser eliminados en un proceso biológico subsecuente (Torres et al., 2003).

La aplicación de la electroquímica al tratamiento de efluentes contaminados con compuestos orgánicos permite reducirlos, transformarlos o eliminarlos (Chen, 2004). Mediante la selección adecuada de los electrodos y del electrolito, se generan durante el proceso electroquímico, oxiradicales con un poder oxidante superior al de cualquier sustancia química oxidante convencional, los cuales dan lugar a la oxidación de la materia orgánica, dando como productos $\mathrm{CO}_{2}$ y $\mathrm{H}_{2} \mathrm{O}$ (Oturan y Brillas, 2007). El uso de electrodo sintético de diamante dopado con boro (BDD) en una oxidación anódica ha demostrado que la sobretensión de $\mathrm{O}_{2}$ es mucho más alta que la observada para los ánodos convencionales tales como $\mathrm{PbO}_{2}, \mathrm{SnO}_{2}$ dopado o $\mathrm{IrO}_{2}$, produciendo mayores cantidades de $\mathrm{OH} \bullet$ a través de la reacción, lo que conlleva a una remoción más rápida y efectiva de los contaminantes. Por lo tanto la oxidación con electrodos BDD parece ser un procedimiento adecuado para mineralizar los compuestos orgánicos (Nars et al, 2009; PalmaGoyes et al., 2010). Las reacciones de generación de especies oxidantes con electrodo BDD se representan con las ecuaciones 5 y 6 :

$$
\begin{array}{ll}
\mathrm{BDD}+\mathrm{H}_{2} \mathrm{O} \rightarrow \mathrm{BDD}+\mathrm{OH}^{\bullet}+\mathrm{H}^{+}+e^{-} & \text {Oxidación anódica } \\
\mathrm{O}_{2}+2 \mathrm{H}^{+}+2 e^{-} \rightarrow \mathrm{H}_{2} \mathrm{O}_{2} & \text { Reducción catódica }
\end{array}
$$

Los resultados de la caracterización de las aguas de lavado de biodiesel, muestran la necesidad de su tratamiento antes de ser vertidas a un cuerpo de aguas, por su alto contenido de Demanda Química de Oxígeno (DQO), Carbono Orgánico Total (COT) y metanol entre otros componentes. Debido a esto, se propone el tratamiento de dichas aguas con tecnologías avanzadas de oxidación no fotoquímicas ozonización y la oxidación electroquímica, cuyos resultados son reportados en el presente trabajo, mostrando como resultado altas remociones de COT, DQO y metanol en las aguas tratadas. 


\section{MATERIALES Y MÉTODOS}

\section{Lavado de Biodiesel}

Con el fin de comparar diferentes parámetros en las tecnologías propuestas en este estudio, se estandarizó a nivel de laboratorio la fabricación y lavado de biodiesel a partir de aceite de palma RBD (Refinado, Blanqueado y Desodorizado). Para la fabricación del biodiesel, se empleó aceite de palma libre de acidez o con una acidez menor a 0,5 mg/g, al cual se le adicionó metanol (99\%, Merck) en una relación molar de 1:6 (aceite:alcohol), en el que se disolvió previamente el catalizador hidróxido de potasio $(\mathrm{KOH}, 85 \%$, Merck) en una cantidad correspondiente al $0.7 \%$ del aceite utilizado. La reacción de transesterificación se realizó a $60^{\circ} \mathrm{C}$ durante una hora y al finalizar la glicerina obtenida fue separada del biodiesel por decantación. El metanol fue recuperado del biodiesel, a $80^{\circ} \mathrm{C}$ en rotaevaporador (R205, Buchi). El biodiesel obtenido fue lavado en etapas, para cada una de ellas se empleó un volumen de agua equivalente al $5 \%$ del volumen de biodiesel lavado y fue adicionada a $60^{\circ} \mathrm{C}$ mientras se burbujeaba aire al biodiesel. El burbujeo fue mantenido durante 5 minutos, luego la mezcla biodiesel-agua se dejó en reposo durante 24 horas y finalmente fue decantada (Fig. 1). Esta etapa de lavado se repitió hasta que el pH del agua decantada fue cercano al neutro o se alcanzó un valor estable para garantizar principalmente la remoción del catalizador.

El agua de lavado empleada en cada una de las etapas fue recolectada para obtener la muestra compuesta. A esta muestra se le retiraron los jabones presentes por medio de una hidrólisis ácida para convertirlos en ácidos grasos de fácil remoción y disminuir la carga orgánica en primera instancia por técnicas físicas, como se realiza a nivel industrial. Posteriormente el agua recolectada fue caracterizada y tratada utilizando las tecnologías avanzadas de oxidación ozonización y oxidación electroquímica.

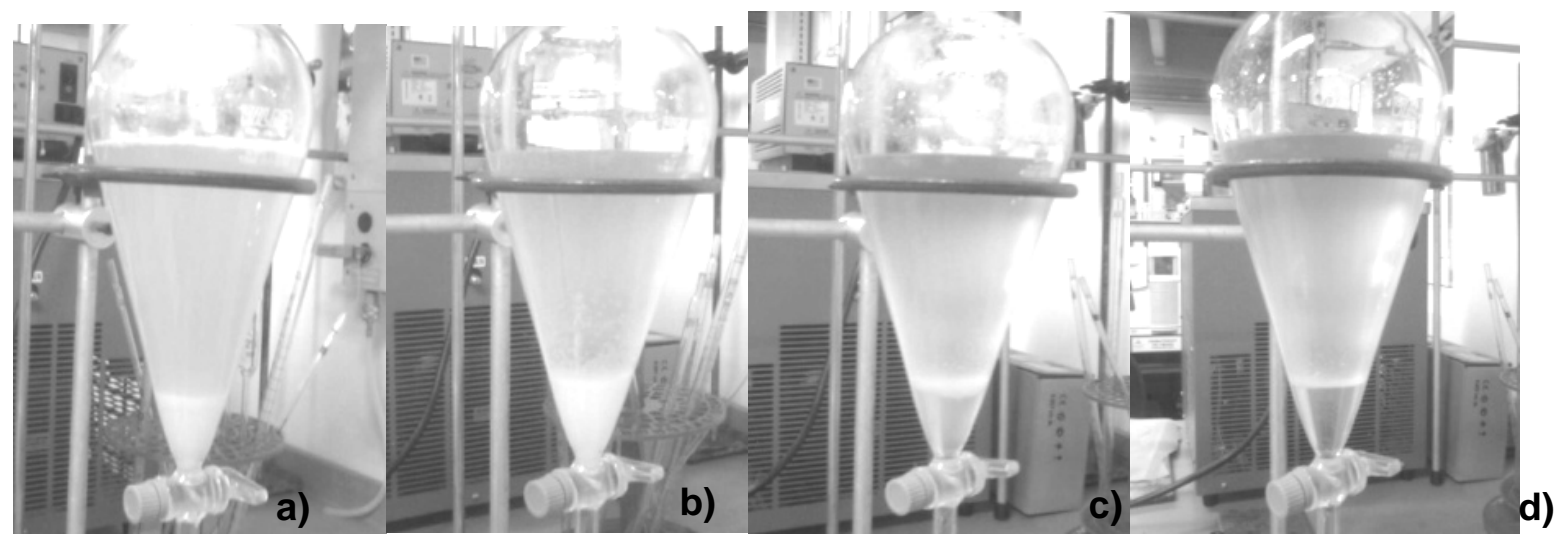

Fig. 1: Imágenes delas etapas del proceso de lavado del biodiesel de palma RBD: a) 24 h, b) 48 h, c) $72 \mathrm{~h}, \mathrm{~d}) 96 \mathrm{~h}$

La caracterización realizada al agua de lavado de la producción de biodiesel y la obtenido luego de los procesos de tratamiento se realizó a través de parámetros normalizados (Standard methods, 2005 y normas DIN), los cuales son mostrados en la Tabla 1. Adicionalmente se determinó el contenido de metanol a través de cromatografía de gases empleando Cromatógrafo GC FID 7890A marca Agilent Technology.

\section{Ozonización}

Se utilizó un ozonizador (Ozonind) con tres diferentes flujos de ozono. El ozonizador fue alimentado directamente con oxígeno grado UPA, garantizando un suministro continuo de oxígeno de $2 \mathrm{~L} / \mathrm{min}$. El agua residual, a temperatura ambiente, se colocó en un reservorio de vidrio de 2 litros de capacidad y el ozono le fue suministrado por medio de un difusor para garantizar la distribución del agente oxidante de forma homogénea. El sistema utilizado para el tratamiento por ozonización, se muestra en la Fig. 2. 
Tabla 1. Parámetros empleados para la caracterización del agua

\begin{tabular}{|c|c|c|c|}
\hline Parámetro & Método de análisis & $\begin{array}{c}\text { Descripción del equipo } \\
\text { utilizado }\end{array}$ & $\begin{array}{l}\text { Método estándar } \\
\text { Norma }\end{array}$ \\
\hline $\mathrm{pH}$ & Potenciométrico & $\begin{array}{l}\text { pH-metro marca WTW, } \\
\text { modelo pH 315i con } \\
\text { electrodo Sentix-41. }\end{array}$ & $\begin{array}{l}\text { Standard Methods } \\
2310-2005\end{array}$ \\
\hline Conductividad & Potenciométrico & $\begin{array}{c}\text { Conductivímetro } \\
\text { Scientific Instrument } \\
\text { IQ350. }\end{array}$ & $\begin{array}{l}\text { Standard Methods } \\
\text { 2510B - } 2005\end{array}$ \\
\hline Turbiedad & Nefelométrico & $\begin{array}{l}\text { Turbidímetro Hach } \\
2100 \mathrm{P} \text {. }\end{array}$ & $\begin{array}{l}\text { Standard Methods } \\
2130 \mathrm{~B}-2005 \\
\end{array}$ \\
\hline DQO & $\begin{array}{l}\text { Reflujo Cerrado, } \\
\text { Espectrofotométrico }\end{array}$ & $\begin{array}{l}\text { Espectrofotómetro UV- } \\
\text { Vis marca Thermo, } \\
\text { modelo evolution } 600 .\end{array}$ & $\begin{array}{c}\text { DIN ISO } 15705 \\
\text { análogo APHA } \\
\text { 5220D } \\
\end{array}$ \\
\hline COT & Infrarrojo & $\begin{array}{l}\text { Espectrofotómetro UV- } \\
\text { Vis marca Thermo, } \\
\text { modelo evolution } 600 .\end{array}$ & $\begin{array}{l}\text { DIN EN1484, } \\
\text { análogo APHA } \\
5310\end{array}$ \\
\hline $\mathrm{DBO}_{5}$ & $\begin{array}{l}\text { Incubación en frascos } \\
\text { Winkler durante } 5 \text { días. }\end{array}$ & $\begin{array}{l}\text { Fotómetro Nanocolor, } \\
\text { marca Macherey-Nagel, } \\
\text { modelo } 500 \text { D. }\end{array}$ & $\begin{array}{c}\text { DIN EN 1899-1- } \\
\text { H51 + DIN EN } \\
25813\end{array}$ \\
\hline Detergentes & $\begin{array}{c}\text { Extracción: determinación } \\
\text { fotométrica con azul de } \\
\text { metileno }\end{array}$ & $\begin{array}{l}\text { Fotómetro Nanocolor, } \\
\text { marca Macherey-Nagel, } \\
\text { modelo } 500 \text { D. }\end{array}$ & $\begin{array}{l}\text { DIN 38409-H23-1 } \\
\text { APHA 5540C }\end{array}$ \\
\hline Alcalinidad & Potenciométrico & $\begin{array}{l}\text { pHmetro marca WTW, } \\
\text { modelo pH } 315 \mathrm{i} \text { con } \\
\text { electrodo Sentix-41. }\end{array}$ & $\begin{array}{l}\text { Standard Methods } \\
\text { 2320B - 2005 }\end{array}$ \\
\hline Color & Espectrofotometría & $\begin{array}{l}\text { Fotómetro Nanocolor, } \\
\text { marca Macherey-Nagel, } \\
\text { modelo } 500 \text { D. }\end{array}$ & $\begin{array}{l}\text { DIN EN ISO } 7887- \\
\text { C1 APHA 2120B }\end{array}$ \\
\hline Toxicidad & $\begin{array}{l}\text { Bioensayos con Daphnia } \\
\text { Pulex }\end{array}$ & $\begin{array}{l}\text { Material Volumétrico de } \\
\text { Laboratorio e } \\
\text { Iluminación }\end{array}$ & $\begin{array}{l}\text { Standard Methods } \\
\quad 8711-2005\end{array}$ \\
\hline
\end{tabular}

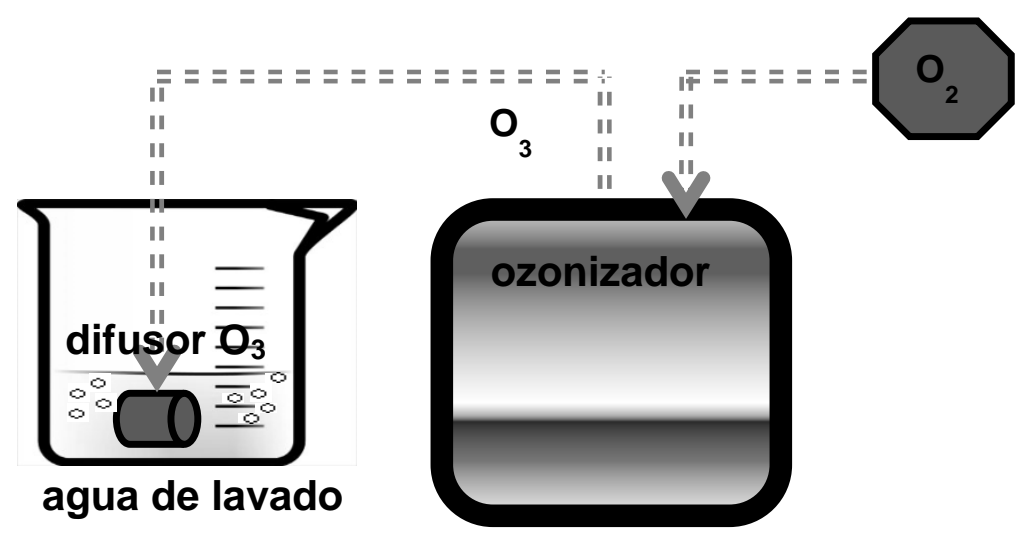

Fig. 2: Esquema del sistema de ozonización compuesto por la fuente de oxígeno, ozonizador (Ozonind), difusor de ozono (Ozonind), recipiente de reacción.

La información reportada para tratamiento de aguas residuales por ozonización (Esplugas, 2002), presentan al flujo de ozono suministrado y el $\mathrm{pH}$ del agua residual a tratar como las principales variables que afectan este proceso (Guinea et al., 2009; Santana et al., 2009), las cuales fueron seleccionadas para el desarrollo de este trabajo. Para llevar a cabo los experimentos, se ajustó el $\mathrm{pH}$ al agua residual con hidróxido de sodio $0.1 \mathrm{~N}$ (Merck) o ácido sulfúrico $0.1 \mathrm{~N}$ (Merck), de acuerdo con el diseño de experimentos, en el cual se varían el pH en tres niveles y el flujo de 
ozono, según la capacidad de trabajo del ozonizador. El diseño de experimentos propuesto, es un diseño $3^{2}$, con tres puntos al centro y se muestra en la Tabla 2 . El pH se midió con un pH-metro portátil 315i (WTW), los análisis de DQO y COT, se realizaron utilizando un fotómetro Nanocolor 500D (Macherey-Nagel) y la medición de metanol se hizo vía SPME-GC-FIT (Agilent, modelo 7890A).

Tabla 2. Diseño experimental para la ozonización

\begin{tabular}{|c|c|c|}
\hline Experimento & $\mathrm{pH}$ & ${\text { Flujo } \mathrm{O}_{3}(\mathrm{~g} / \mathrm{h})}$ \\
\hline 1 & 4,5 & 0,34 \\
\hline 2 & 7,0 & 0,34 \\
\hline 3 & 12,0 & 0,34 \\
\hline 4 & 12,0 & 0,26 \\
\hline 5 & 7,0 & 0,26 \\
\hline 6 & 4,5 & 0,26 \\
\hline 7 & 4,5 & 0,17 \\
\hline 8 & 7,0 & 0,17 \\
\hline 9 & 12,0 & 0,17 \\
\hline 10 & 7,0 & 0,26 \\
\hline 11 & 7,0 & 0,26 \\
\hline 12 & 7,0 & 0,26 \\
\hline
\end{tabular}

\section{Oxidación Electroquímica}

Los ensayos de oxidación electroquímica se realizaron utilizando una celda electroquímica con capacidad de $250 \mathrm{~mL}$, provista de cuatro bocas para toma de muestras, medición de temperatura y suministro de voltaje, con agitación magnética constante y con un electrodo de diamante dopado con boro soportado sobre silicio (BDD-Adamant Technologies con un espesor de $3 \mu \mathrm{m}$ ) como ánodo, el cual posee un área de contacto de $4 \mathrm{~cm}^{2}$, mientras que el cátodo utilizado fue un espiral de zirconio. El sistema fue alimentado utilizando una fuente de corriente directa (BK Precision), fijando valores entre 0,01 y 0,04 A, de acuerdo con el diseño de experimentos. Al inicio de cada prueba, se adicionó la cantidad respectiva de electrolito, cloruro de sodio, 99,5\% Merck, según el caso. Los análisis de DQO y COT, al igual que para los experimentos de ozonización, se realizaron utilizando un fotómetro Nanocolor 500D (Macherey-Nagel) y la medición de metanol se hizo vía SPME-GC-FIT (Agilent, modelo 7890A). El sistema completo de electroquímica se muestra en la Fig. 3.

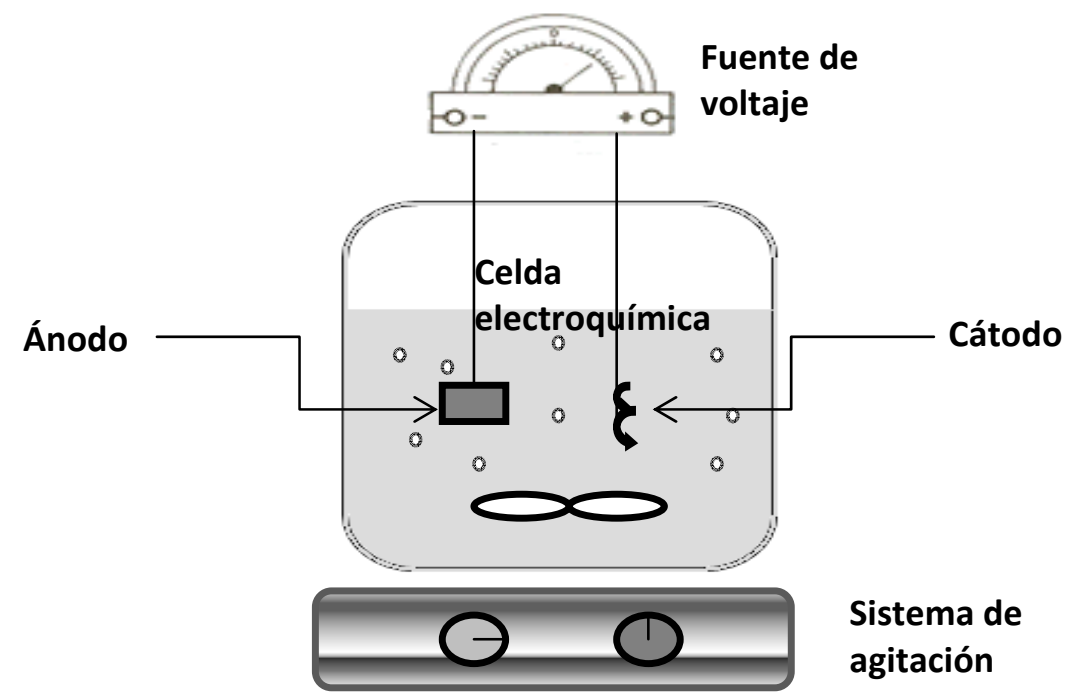

Fig. 3: Esquema del sistema de oxidación electroquímica compuesto por la celda electroquímica, ánodo de BDD-Adamant, cátodo de zirconio, sistema de agitación y fuente de corriente BK 
De acuerdo con la información encontrada para tratamiento de aguas residuales por electroquímica, se ha observado una gran influencia de la concentración del electrolito y de la densidad de corriente suministrada al sistema.Se aplicó entonces un diseño de experimentos en el cual se varían la concentración de electrolito, en este caso el cloruro de sodioen cinco niveles y la densidad de corriente en cuatro niveles (Cañizares et al., 2009). El diseño de experimentos propuesto, es un diseño de cara centrada, con tres puntos al centro y se muestra en la Tabla 2.

Tabla 3. Diseño experimental cara centrada con tres puntos al centro para la oxidación electroquímica

\begin{tabular}{|c|c|c|}
\hline Experimento & Densidad de corriente (A) & Concentración de electrolito (g/L) \\
\hline 1 & 0,03 & 0,69 \\
\hline 2 & 0,03 & 1,75 \\
\hline 3 & 0,04 & 2,5 \\
\hline 4 & 0,03 & 2,81 \\
\hline 5 & 0,03 & 1,75 \\
\hline 6 & 0,04 & 1,75 \\
\hline 7 & 0,03 & 1,75 \\
\hline 8 & 0,02 & 1,0 \\
\hline 9 & 0,04 & 1,0 \\
\hline 10 & 0,02 & 2,5 \\
\hline 11 & 0,01 & 1,75 \\
\hline
\end{tabular}

\section{RESULTADOS Y DISCUSIÓN}

Caracterización de las aguas de lavado de biodiesel de palma

Los resultados de la caracterización del agua de lavado de biodiesel de palma se muestran en la Tabla 4. Los altos valores de los parámetros medidos, muestran que el agua residual requiere de un tratamiento de descontaminación antes de ser descargada a cualquier efluente. A pesar de que se realizó un proceso previo de recuperación del metanol el agua de lavado aún contiene dicho compuesto, lo cual contribuye significativamente a la toxicidad de las aguas.

Tabla 4. Caracterización del agua de lavado de biodiesel de Palma con catalizador $\mathrm{KOH}$

\begin{tabular}{|c|c|}
\hline Parámetros & Valor \\
\hline $\mathrm{pH}$ & 11,11 \\
\hline Conductividad $(\mu \mathrm{S})$ & 758 \\
\hline Turbiedad $(\mathrm{NTU})$ & $>1000$ \\
\hline $\mathrm{DQO}(\mathrm{mg} / \mathrm{L})$ & 17750 \\
\hline $\mathrm{COT}(\mathrm{mg} / \mathrm{L})$ & 6620 \\
\hline DBO $_{5}\left(\mathrm{mg} / \mathrm{L} \mathrm{O}_{2}\right)$ & 7980 \\
\hline Detergentes $(\mathrm{ppm}))$ & 1883,3 \\
\hline Alcalinidad $\left(\mathrm{mg} / \mathrm{L} \mathrm{CaCO}_{3}\right)$ & $>500$ \\
\hline Color $(\mathrm{Hazen})$ & 3,15 \\
\hline \%Metanol & 100 \\
\hline Toxicidad en Daphnia Pulex. $(\%)$ & \\
\hline
\end{tabular}




\section{Ozonización}

El diseño de experimentos planteado fue aplicado sobre una matriz con una carga orgánica cien veces menor a la descarga extraída del proceso con el fin de evaluar los parámetros propios de la tecnología. Se obtuvieron remociones altas de COT y DQO a esta dilución de agua de lavado (Fig. 4).

Los resultados que produjo el diseño de experimentos muestran que la concentración de ozono es la variable que más influye en el proceso evaluado, ya que se dispone de mayor cantidad de oxidante para reaccionar con los compuestos orgánicos presentes en el agua y por lo tanto para un tiempo de degradación fijo la eficiencia de la reacción es mejor cuando se dispone de mayor cantidad de oxidante. Las condiciones del sistema más adecuadas dentro del rango de evaluación del diseño de experimentos fueron: flujo de ozono $0.34 \mathrm{~g} / \mathrm{h} \mathrm{y} \mathrm{pH}$ de 12 , con las cuales se alcanzaron remociones de $79,41 \%$ para COT y de $81,20 \%$ para DQO. Es importante resaltar el experimento $6(0,26 \mathrm{~g} / \mathrm{h}$ de ozono y $\mathrm{pH}=4,5)$, con un valor de $\mathrm{pH}$ en nivel bajo y flujo de ozono intermedio, en el cual se obtuvo alto porcentaje de remoción de los parámetros COT y DQO, condiciones que resultan más económicas en el caso de realizar un escalado del proceso. De acuerdo con el análisis estadístico, realizado empleando el programa Statgraphics, el pH no es una variable significativa, lo cual es importante ya que en aplicaciones a mayor escala no sería necesario su ajuste o modificación, lo que haría aún más atractiva la tecnología. Sin embargo se observó que a un pH de 12,0, se obtuvieron los mejores resultados, debido a que la alcalinidad de la solución acelera la formación de radicales hidroxilo activos por la interacción del ozono con el ion hidróxido.

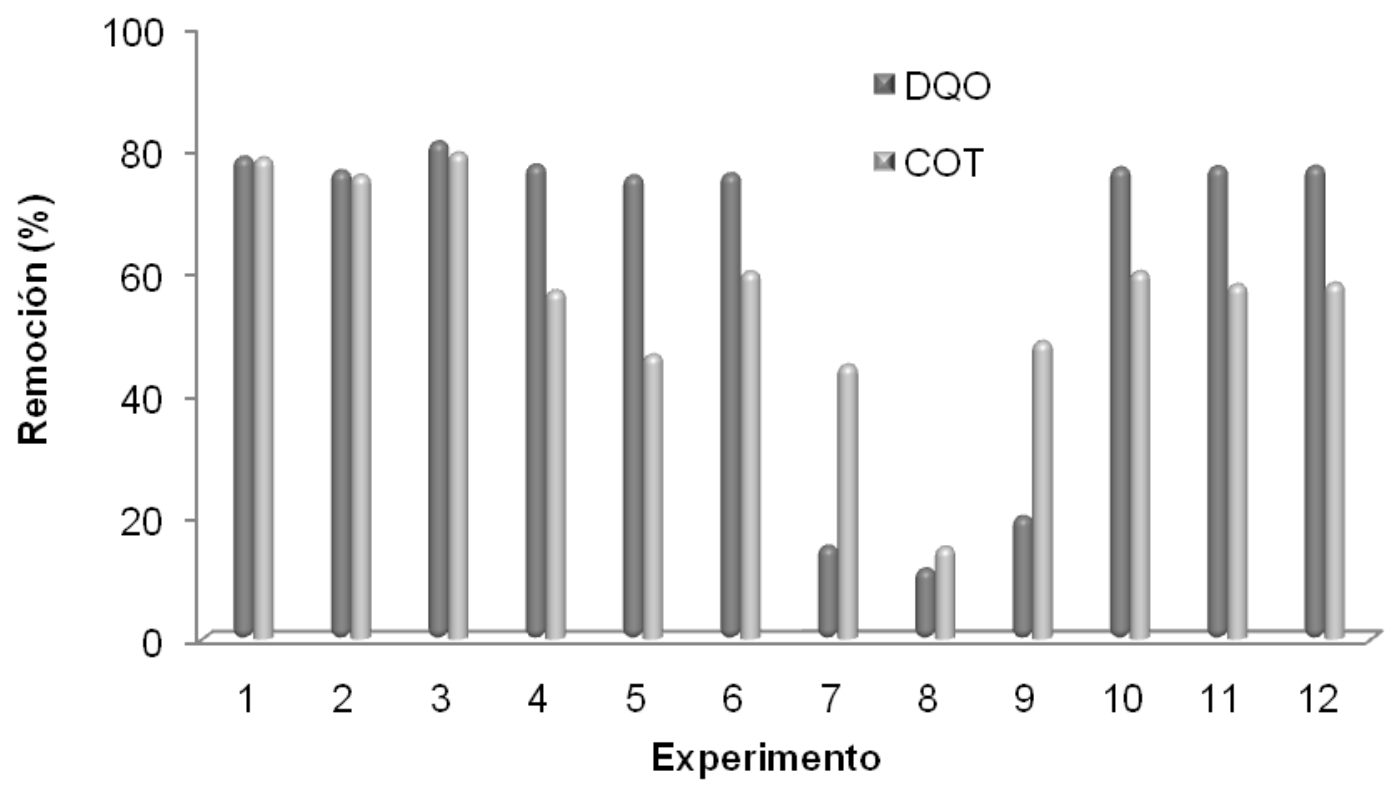

Fig. 4: Porcentajes de remoción de DQO y COT en aguas de lavado de biodiesel de concentración cien veces menor a la extraída del proceso con tecnología de ozonización.

El arreglo de mejor respuesta, correspondiente al ensayo 3 (flujo de ozono de 0,34 g/h y un pH de 12,0 ), fue aplicado a una matriz de aguas residuales de biodiesel, con una dilución cinco veces menor a la descarga original extraída del proceso de lavado. Las remociones observadas, fueron inferiores a las obtenidas para la dilución de cien veces, con valores de remoción en COT y DQO de 22 y $21 \%$, además fue determinado el porcentaje de remoción del metanol residual, el cual se ubicó en el 31\%, Fig. 5. Estos resultados muestran que la tecnología todavía presenta una eficiencia significativa a pesar de tratar una carga contaminante 20 veces más alta.

El arreglo seleccionado se probó finalmente con el agua residual sin ningún tipo de dilución, con el fin de tener datos similares a los que se obtendrían con las aguas de tipo industrial. El porcentaje 
de remoción de materia orgánica fue menor comparado con los resultados obtenidos sobre la dilución anterior. No obstante, a pesar de las altas concentraciones la tecnología todavía presenta una respuesta de tratamiento positivo sobre las aguas residuales de la producción de biodiesel alcanzando remociones del 12 y del $14 \%$ para el COT y el metanol respectivamente, mientras que para del DQO, la reducción es solo de aproximadamente el 5\%, Fig. 5.

Se realizó un análisis de biodegradabilidad de las muestras de agua, antes y después del proceso de tratamiento, determinando la relación $\mathrm{DBO}_{5} / \mathrm{DQO}$. Este análisis muestra que a pesar de los porcentajes de degradación logrados, la relación pasó de 0.45 a 0.49 , lo que incrementó la biodegrabilidad de muestra y haciéndola más apta para su tratamiento empleando procesos como los biológicos.

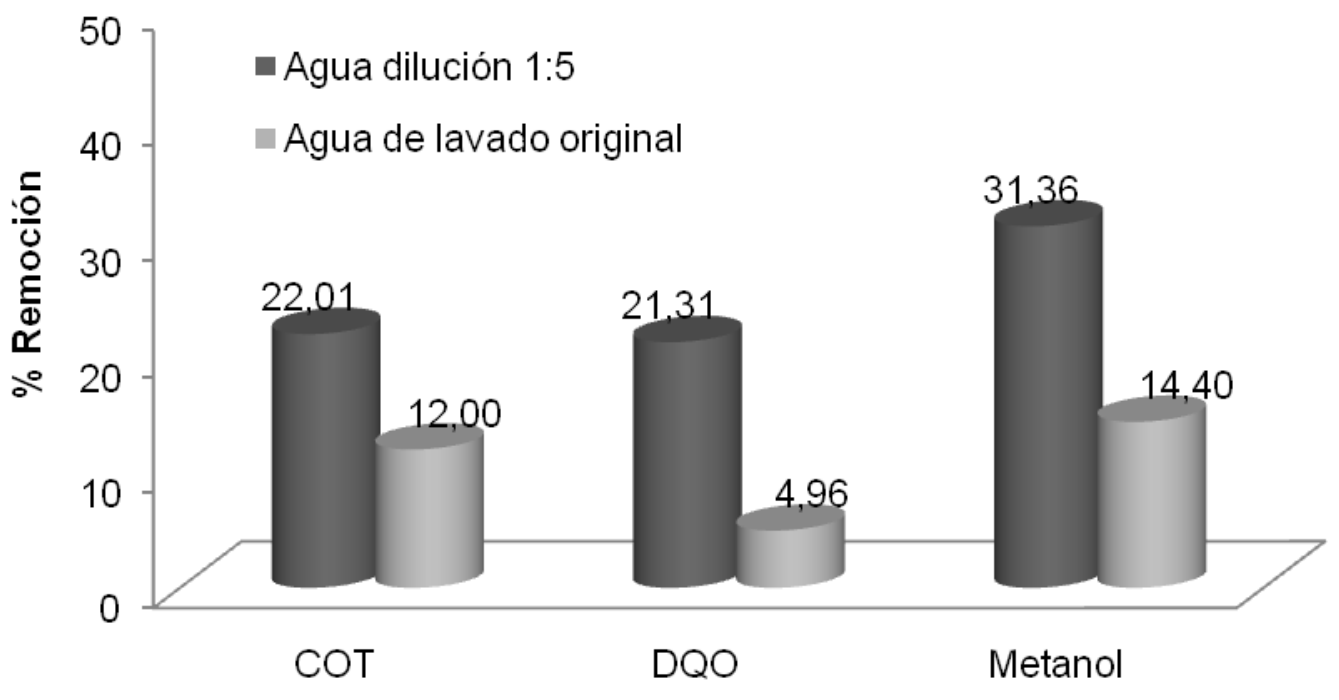

Fig. 5: Porcentajes de remoción de DQO y COT y metanol en aguas de lavado de biodiesel de concentración cinco veces menor y directamente extraída del proceso con tecnología de ozonización (flujo de ozono de 0,34 g/h y un pH de 12,0).

\section{Electroquímica}

La Fig. 6 muestra los porcentajes de remoción de DQO y COT del diseño de experimentos aplicado sobre una carga orgánica cien veces menor a la descarga extraída del proceso con el fin de evaluar los parámetros propios de la tecnología.

En el caso delatecnología de oxidación electroquímica, la mayor influencia en la remoción de contaminantes la presentó la concentración de electrolito. En la mayoría de los valores de corriente aplicados se observan remociones del orden del 40\%, tanto en DQO como en COT, usando una concentración de electrolito alta. De acuerdo con el análisis estadístico determinado con el programa Statgraphics, la corriente aplicada no es significativa en la remoción de DQO y en consecuencia para una concentración de electrolito alta, es suficiente aplicar una corriente baja para obtener resultados de remoción satisfactorios con una disminución en el gasto final de energía.

Las remociones más altas, 58,49\% en DQO y un 61,11\% en COT, fueron logradas empleando como parámetros de reacción una corriente de 0,04 A y una concentración de electrolito de 2,5 g/L de $\mathrm{NaCl}$. Estas condiciones fueron aplicadas sobre cargas orgánicas más altas, en este caso, una carga 5 veces menor a la extraída y la directamente obtenida del proceso de lavado de biodiesel, cuyos resultados se muestran en la Fig. 7. Se observa la disminución en el porcentaje de remoción de COT y DQO así como en el contenido de metanol. No obstante, la tecnología muestra aún tener una respuesta positiva sobre las aguas residuales de la producción de 
biodiesel, principalmente en la remoción de metanol, el cual muestra incluso valores similares a los obtenidos cuando se tratan aguas con una carga cinco veces menor. Las remociones logradas son, en magnitud, similares a las alcanzadas con la tecnología de ozonización en las aguas en concentración completa, sin embargo el aumento de biodegradabilidad, determinado como $\mathrm{DBO}_{5} / \mathrm{DQO}$, logrado es menor ya que solo se pasa de 0.45 a 0.47 aunque este pequeño aumento podría ser importante en sistemas acoplados.

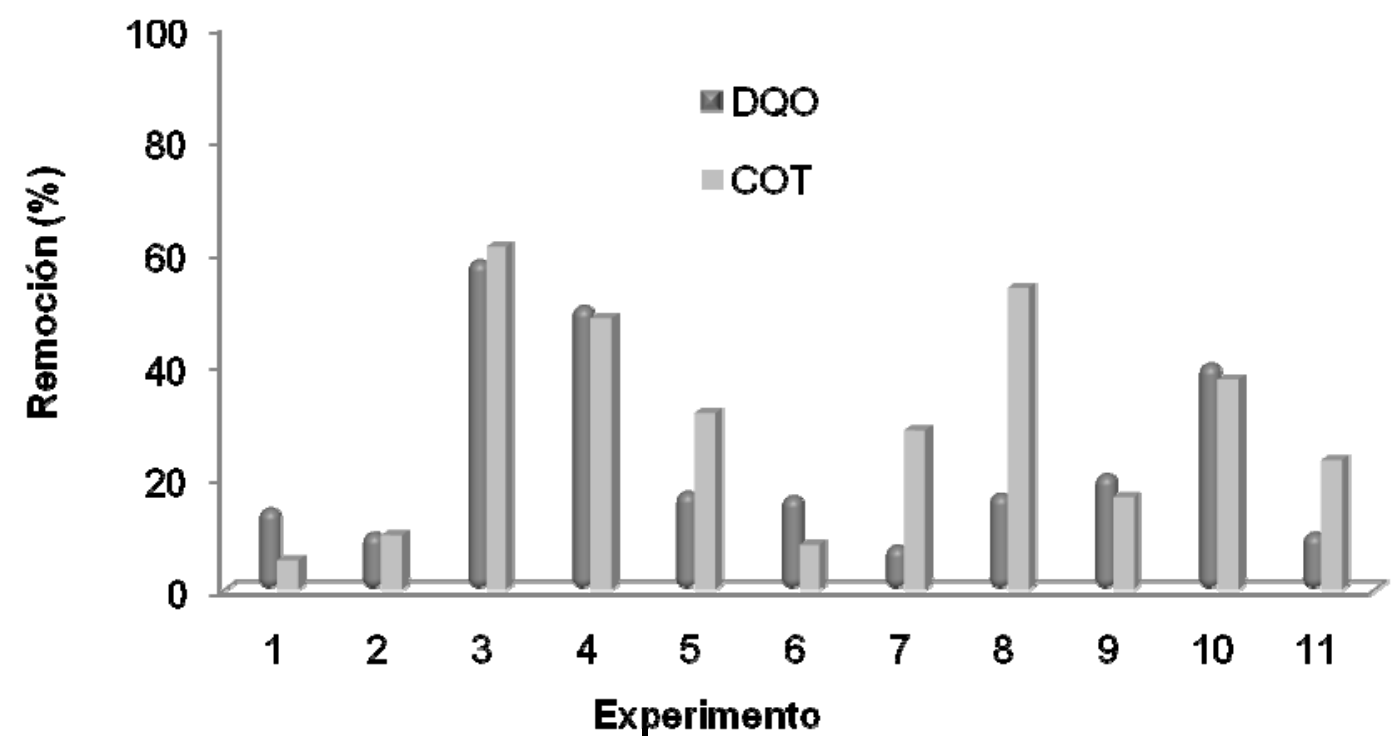

Fig. 6: Porcentajes de remoción del Carbono Orgánico Total y Demanda Química de Oxígeno en aguas de lavado de biodiesel de concentración cien veces menor a la extraída del proceso con la tecnología de oxidación electroquímica

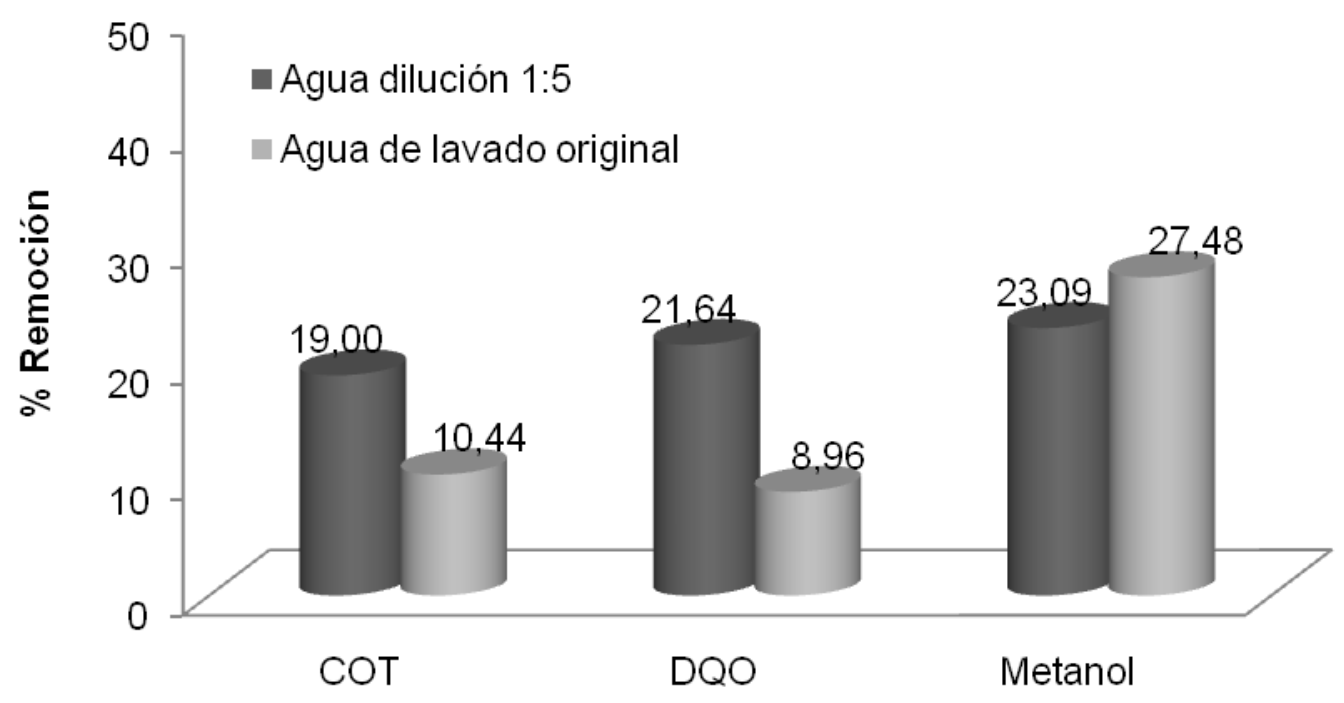

Fig. 7: Porcentajes de remoción de COT, DQO y Metanol en aguas de lavado de biodiesel de concentración cinco veces menor y directamente extraída del proceso con la tecnología electroquímica $([\mathrm{NaCl}]=2,5 \mathrm{~g} / \mathrm{L}$ y $0,04 \mathrm{~A})$ 


\section{CONCLUSIONES}

Las remociones encontradas con la tecnología ozonización son en general similares a las alcanzadas con la tecnología de oxidación electroquímica, sin embargo, la tecnología de ozonización presenta características técnicas que permiten un escalado más fácil ya que puede utilizarse aire del ambiente para la producción de ozono, por medio de un compresor o un ventilador y adicionalmente degrada los contaminantes en un rango amplio de $\mathrm{pH}$ y flujo de ozono. La condición encontrada como óptima en este proceso fue un flujo de ozono de $0,34 \mathrm{~g} / \mathrm{h}$ y un $\mathrm{pH}$ de 12,0, sin embargo, la tecnología muestra también buenos valores de remoción a pH neutro, lo que facilitaría su manejo y reduciría los costos para una implementación práctica.

Las tecnologías de ozonización y oxidación electroquímica podrían ser empleadas para el tratamiento de las aguas de lavado de la producción de biodiesel como tratamientos previos a un proceso biológico ya que aumenta la biodegradabilidad de esta clase de agua residual al disminuir las cargas contaminantes, especialmente el contenido de metanol, en corto tiempo, posibilitando el tratamiento con microorganismos.

\section{AGRADECIMIENTOS}

Los autores agradecen al 'Ministerio Colombiano de Agricultura y Desarrollo Rural' y a la Universidad de Antioquia por la financiación de este proyecto enmarcado dentro del programa 'Nuevas Tecnologías para mejorar la producción de biodiesel desde el punto de vista tecnológico, económico y ambiental' Nº 017-2008D3873-4049.

\section{REFERENCIAS}

Beltrán, F., Ozone Reaction Kinetics for Water and Wastewater Systems, $1^{a}$ edición, 1-358. Lewis Publishers, New York, Estados Unidos (2004)

Berrios, M y R.L. Skelton, Comparison of purification methods for biodiesel, Chemical Engineering Journal: 144(3), 459-465 (2008).

Brillas, E. y otros seis autores, Mineralization of paracetamol in aqueous medium by anodic oxidation with a boron-doped diamond electrode, Chemosphere: 58(4), 399-406 (2005).

Cañizares, P. y otros cinco autores, A comparison between Conductive-Diamond Electrochemical Oxidation and other Advanced Oxidation Processes for the treatment of synthetic melanoidins, Journal of Hazardous Materials: 164(1), 120-125 (2009).

Chen, G., Electrochemical technologies in wastewater treatment, Separation and Purification Technology: 38(1), 11-41 (2004).

Domènech, X., W.F. Jardim y M.I. Litter, Procesos avanzados de oxidación para la eliminación de contaminantes, Eliminación de contaminantes por fotocatálisis heterogénea, $1^{\text {a }}$ edición, CIEMAT, pp. 3-26, Madrid, España (2004).

Esplugas, S., J. Giménez, S. Contreras, E. Pascual y M. Rodríguez, Comparison of different advanced oxidation processes for phenol degradation, Water Research, 36(4), 1034-1042 (2002).

Guillard, C., E. Puzenat, H. Lachheb, A. Houas, A. y J.M. Herrmann, Why inorganic salts decrease the $\mathrm{TiO}_{2}$ photocatalytic efficiency, International Journal of Photoenergy: 7(1), 1-9 (2005).

Guinea E. y otros cinco autores, Oxidation of enrofloxacin with conductive-diamond electrochemical oxidation, ozonation and Fenton oxidation. A comparison, Water Research: 43(8), 2131-2138 (2009). 
Habib, Z., R. Parthasarathy y S. Gollahalli, Performance and emission characteristics of biofuel in a small-scale gas turbine engine, Applied Energy: 87(5), 1701-1709 (2010).

Hincapié-Mejía, G.M., D. Ocampo, G. Restrepo, y J.M. Marín, Fotocatálisis Heterogénea y FotoFenton Aplicadas al Tratamiento de Aguas de Lavado de la Producción de Biodiesel, Información tecnológica: 22(2), 33-42 (2011).

Komers, K., F. Skopal y A. Cegan, Continuous biodiesel production in a cascade of flow ideally stirred reactors, Bioresource Technology: 101(10), 3772-3775 (2010).

Nasr, B., T. Hsen y G. Abdellatif, Electrochemical treatment of aqueous wastes containing pyrogallol by BDD-anodic oxidation, Journal of Environmental Management: 90(1), 523-530 (2009).

Oturan, M.A. y E. Brillas, Electrochemical Advanced Oxidation Processes (EAOPs) for Environmental Applications, Portugaliae Electrochimica Acta: 25, 1-18 (2007).

Palma-Goyes, R. E. y otros cinco autores, Electrochemical degradation of crystal violet with BDD electrodes: Effect of electrochemical parameters and identification of organic by-products, Chemosphere: 81(1), 26-32 (2010).

Robinson, T., G. McMullan, R. Marchant y P. Nigam, Remediation of dyes in textile effluent: a critical review on current treatment technologies with a proposed alternative, Bioresource Technology: 77(3), 247-255 (2001).

Santana M. y otros cinco autores, Application of electrochemically generated ozone to the discoloration and degradation of solutions containing the dye Reactive Orange 122, Journal of Hazardous Materials: 164(1), 10-17 (2009).

Srirangsan, A., M. Ongwandee y O. Chavalparit, Treatment of biodiesel wastewater by electrocoagulation process, Environment Asia: 2(2), 15-19 (2009)

Suehara, K.I. y otros cinco autores, Biological Treatment of Wastewater Discharged from Biodiesel Fuel Production Plant with Alkali-Catalyzed Transesterification, Journal of Bioscience and Bioengineering: 100(4), 437-442 (2005)

Torres, R.A., V. Sarria, W. Torres, P. Péringer y C. Pulgarín, Electrochemical treatment of industrial wastewater containing 5-amino-6-methyl-2-benzimidazolone: toward an electrochemicalbiological coupling, Water Research: 37(13), 3118-3124 (2003). 